\title{
Un modelo de atención en rehabilitación basado en la bioética para personas en situación de discapacidad
}

\author{
Silvia Cristina Duarte Torres*
}

\begin{abstract}
Resumen
Según cifras de Naciones Unidas y Asociaciones Internacionales, existen entre 500 y 600 millones de personas en el mundo que presentan algún tipo de discapacidad física o mental, es decir, un 10\% de los habitantes del mundo. Además, se estima que el $25 \%$ de la población total se ve afectada por las dificultades existentes para las personas con discapacidad. Asimismo, Naciones Unidas señala que un $98 \%$ de las personas con discapacidad viven en países en desarrollo, que no tienen acceso a servicios de rehabilitación, que sólo un $25 \%$ de las personas cuenta con algún empleo o ingreso, dicho de otra forma, el $75 \%$ restante es considerado inactivo. Más aún, el $80 \%$ depende de sus familiares o amigos y cerca del 10\% vive de la caridad o limosna. El proceso de rehabilitación involucra directamente los principios éticos como la defensa de la dignidad de las personas, el servicio, la solidaridad entre otros. Este tema de la atención de la persona con discapacidad se relaciona directamente con la bioética, actual disciplina que reformula una ética de la vida para los tiempos de alta tecnología. Este artículo es una reflexión sobre los principales dilemas de tipo bioético de los profesionales de la rehabilitación, los diferentes modelos de rehabilitación que se han desarrollado a través del tiempo y finalmente se establece una propuesta de modelo de atención para la persona con discapacidad basado en la bioética.
\end{abstract}

Palabras clave: bioética, rehabilitación, discapacidad.

\section{INTRODUCCIÓN}

La rehabilitación nace como respuesta de la sociedad, y específicamente de la medicina para las personas con discapacidad, combinando conocimientos y técnicas interdisciplinarias susceptibles de mejorar el pronóstico funcional de estos seres humanos. Este conjunto organizado de actividades, procedimientos e intervenciones tendientes a desarrollar, mejorar, mantener o restaurar la capacidad funcional, previene, modifica, aminora o desaparece las consecuencias de la edad, la enfermedad o los accidentes. Una fase final de la rehabilitación es la profesional que incluye la evaluación, orientación, capacitación y ubicación laboral. Hasta hace poco la persona con discapacidad se estimaba como un problema exclusivamente clínico, pero en la actualidad es con-

* Terapeuta ocupacional. Magistra en Bioética y Decana de la Facultad de Terapia Ocupacional de la Escuela Colombiana de Rehabilitación. 
siderada como un problema de salud pública y de impacto social. Los deberes y derechos de la población con limitaciones físicas, mentales y/o sensoriales se soportan en normas, convenios y declaraciones internacionales que se van desarrollando acorde con los enfoques de atención y reconocimiento de las personas con discapacidad.

La rehabilitación es un proceso global y continuo de duración limitada y con objetivos definidos; la rehabilitación pretende facilitar la vida de una persona que con deficiencia o discapacidad alcance un nivel funcional óptimo, proporcionándole los medios que le posibiliten llevar en forma independiente y libre su vida.

No cabe duda, que la legislación internacional y colombiana existente hasta el momento es amplia y adecuada, sin embargo, el problema continúa aumentando y la calidad de vida de las personas no ha mejorado de acuerdo a lo esperado. En los marcos políticos nacionales e internacionales se encuentra que la atención de las personas con discapacidad está favorecida, haciendo posible un desarrollo de actitudes y acciones humanas responsables y tolerantes.

En general los antecedentes en rehabilitación no reportan una infraestructura clara acerca de cómo intervienen los aspectos bioéticos en la creciente complejidad de los servicios de rehabilitación y la atención a las personas con discapacidad, teniendo en cuenta el desarrollo científico, técnico y las condiciones de la economía actual en el mundo. Estos aspectos se consideran un reto para todos los participantes en este proceso a fin de introducir una cultura de la eficiencia. En la práctica de la rehabilitación a menudo los principios bioéticos de beneficencia, no maleficencia, decir la verdad al paciente, autonomía y justicia entran en conflicto debido a la falta de recursos, a los costos, la falta de conciencia de querer mejorarse y los problemas de índole administrativo, estando involucrada de forma significativa la responsabilidad social.

\section{Discapacidad y rehabilitación}

La vida de las personas con limitaciones físicas, mentales y sensoriales, su situación social y económica, así como el trato discriminado que reciben han variado en los diferentes grupos y épocas de acuerdo con las actitudes sociales, grados de cultura y conceptos morales. Durante siglos han sido discriminadas, despreciadas o compadecidas, en general, temidas e incomprendidas.

En términos de las respuestas sociales frente a las personas con discapacidad es posible identificar algunas posturas que permiten comprender los conceptos que se han afianzado en la cultura a lo largo de la historia (Tabla 1). Estos conceptos se configuran a partir de explicaciones, ya sean teóricas, intuitivas o del sentido común que expresan cómo la sociedad enfrenta la condición de insuficiencia y discapacidad.

\section{Tabla 1}

Discapacidad a tRaVÉs de la historia

\begin{tabular}{|c|c|}
\hline ÉPOCA & $\begin{array}{l}\text { ACTITUD FRENTE A LA } \\
\text { PERSONA CON } \\
\text { DISCAPACIDAD }\end{array}$ \\
\hline Edad Antigua & Eliminación física \\
\hline Edad Media & Endiosamiento \\
\hline Renacimiento & Protección \\
\hline Edad Moderna & Ocultamiento vergonzante \\
\hline Industrialización & $\begin{array}{l}\text { Reflexión cognoscitiva y } \\
\text { negación fallida }\end{array}$ \\
\hline Siglo XIX & Existencialismo \\
\hline Siglo XX & Rehabilitación \\
\hline
\end{tabular}

En cada uno de los momentos de la historia la actitud de las personas con limitaciones, etiquetadas como minusválidos, impedidos, inválidos o limitados, ha sido de total sometimiento y dependencia, siempre han asumido un papel de rechazo y muerte por considerárseles expresión de 
mal. Este rechazo con el tiempo se convirtió en sobreprotección, y luego, en objetos de caridad; más tarde, en objetos de asistencia, para irse transformando poco a poco en objetos de estudio.

A través de la evolución de la humanidad se identifican tres formas de comportamiento social frente a las personas con limitaciones:

- Rechazo, por estar frente a un fenómeno desconocido y amenazador.

- Protección, por considerarlos incapaces de valerse por sí mismos.

- Justicia social, en la cual todos los miembros que conforman una sociedad se consideran autónomos y participan en igualdad de oportunidades.

En la actualidad la persona en situación de discapacidad es considerada con derechos y deberes, vista desde una perspectiva ecológica (interacción - persona - ambiente). De este modo, en 1997 la Organización Mundial de la Salud definió la discapacidad de una persona como el resultado de la interacción de sus capacidades y las variables ambientales de carácter físico, las situaciones sociales y los recursos. Dentro del modelo funcional de la discapacidad la limitación es una falta o anormalidad del cuerpo de una función fisiológica o psicológica; la actividad es la naturaleza y amplitud del funcionamiento a nivel personal; y la participación es la naturaleza y la amplitud de la implicación de una persona en las situaciones de la vida relacionadas con las limitaciones, actividades, condiciones de salud y factores contextuales.

De la historia de la rehabilitación, no existe información escrita que permita hacer un recuento fiel de lo que ha sido su evolución. Las primeras referencias escritas aparecen en la edad contemporánea, donde la palabra Rehabilitación, deriva del latín medieval y significa literalmente, vuelta a la buena salud, sin embargo, la mayoría de los historiadores han señalado que la palabra rehabilitación fue adoptada para designar el nue- vo servicio gestado a partir de las desastrosas consecuencias de la Primera Guerra Mundial (Bauer, 1992) . Desde entonces la rehabilitación ha estado sujeta a controversia por la lucha llevada a cabo por las personas afectadas de incapacidades y por los profesionales que brindan este servicio.

La rehabilitación por lo tanto, debe ser vista como un medio, más no como un fin pues su objetivo es crear las condiciones para que la persona con limitaciones sea ella misma, se transforme y el entorno le facilite la autonomía y la equiparación de oportunidades.

Por esto se hace necesario conocer cuáles son los enfoques que han orientado los modelos de atención en rehabilitación. Los modelos de atención en rehabilitación son:

- el tradicional o médico

- el de vida independiente y

- el basado en comunidad

Los tres modelos desarrollados en rehabilitación hasta el momento han tenido una evolución acorde a la historia de la humanidad y de la salud. La participación de las personas con discapacidad ha ido en ascenso y la participación de los profesionales de la rehabilitación se ha disminuido. La sociedad, cada vez, tiene una mayor responsabilidad con las personas en situación de discapacidad y si se analiza de acuerdo con los principios, el modelo tradicional gira alrededor de la beneficencia, el de vida independiente alrededor de la autonomía y el de rehabilitación comunitaria en el de justicia.

El modelo de rehabilitación tradicional se desarrolla a partir de 1945 donde la persona con discapacidad toma un rol pasivo y el profesional en rehabilitación una actitud paternalista. 
El modelo de rehabilitación de vida independiente, nace alrededor de 1960-1970, donde la persona en situación de discapacidad toma un rol más activo y desarrollan en conjunto con los profesionales en rehabilitación un proceso democrático.

El modelo de rehabilitación basado en la comunidad se establece en 1990, donde la persona en situación de discapacidad desarrolla un rol activo y participativo, así como los profesionales en rehabilitación. Actualmente, este último modelo es el que se ha venido estableciendo en toda Latinoamérica.

\section{Bioética, discapacidad y rehabilitación}

Al relacionar la bioética con la discapacidad y la rehabilitación se pretende hacer un acercamiento entre los conflictos de los derechos de las personas, el sentido de la rehabilitación y las limitaciones que presenta un ser humano en una situación de discapacidad.

La propuesta de Beauchamp y Childress en Principles, se inspira en el informe Belmont (1979), desarrollando y profundizando sobre la bioética basada en los principios. Aunque ellos niegan la existencia de un orden lexicográfico entre los principios, no cabe duda que el respeto por la autonomía juega un papel central en la propuesta Ferrer (1999). Los autores examinan el concepto de autonomía en el contexto de toma de decisiones, se proponen encontrar una comprensión de la autonomía que permita determinar el campo de acción humano amparado por las normas éticas y legales del consentimiento informado, el derecho a rechazar tratamientos, los deberes relacionados con la obligación profesional de guardar el secreto o decir la verdad sobre su futura calidad de vida para la persona con discapacidad.

Es importante precisar que no es lo mismo ser autónomo que ser respetado como un sujeto autónomo. El respeto por la autonomía exige, en primer lugar, que se reconozca el derecho del sujeto moral, de la persona que es capaz de decidir autónomamente, a tener sus propios puntos de vista; en segundo lugar, tener varias opciones, y por último, obrar en conformidad con sus valores y creencias.

La Asamblea General de las Naciones Unidas, proclamó la declaración Universal de los Derechos Humanos (1948) donde se consigna la igualdad y la dignidad de los seres humanos, constituyéndose en un sólido muro de contención; se dirige a ofrecer a los individuos condiciones de vida social con las cuales les sea posible desarrollarse libremente mediante el uso de facultades naturales, y en particular la libertad, condición propiamente humana que implica pensar y simbolizar.

Los derechos de las personas con discapacidad tienen como lema "derechos, no caridad, respeto y no piedad". Los grupos de personas impedidas desarrollan unos derechos de acuerdo a los principios de igualdad, diversidad, equidad, universalidad, autonomía, vida independiente, equiparación de oportunidades, alternativas de integración y normalización, calidad de vida y protección integral.

Lo que se puede concluir es que cada día contamos con un número mayor de personas con discapacidad que ni siquiera tienen un diagnóstico veraz de su limitación y por lo tanto no saben cómo acceder a la atención en rehabilitación. De acuerdo a un estudio piloto realizado por la Pontificia Universidad Javeriana en 1994, solamente el $15 \%$ de la población con discapacidad tiene acceso a un servicio integral de rehabilitación.

Se hace necesario especificar la aplicación de los principios para ver la viabilidad de la propuesta, teniendo en cuenta cada uno de los factores que intervienen: la cultura, las normas jurídicas, el sistema de salud, entre otras, las cuales influyen directamente en el proceso de rehabilitación. 
Es asi, que en una situación de atención a la persona con discapacidad, para iniciar un proceso deliberativo es necesario aplicar los principios de autonomía y justicia, los cuales permiten resolver un conflicto de calidad de vida (maximizar el bien de la situación), siendo la bioética principialista la escogida para resolver el conflicto de la persona con discapacidad.

\section{Modelo de atención basado en la bioética}

La presentación del modelo de rehabilitación basado en la bioética está estructurada en la aplicación de los principios bioéticos dentro del proceso integral, donde el principio que rige la intervención es el de la autonomía. La persona con discapacidad debe conocer que tiene el derecho y el deber de hacer uso de su autonomía y este hecho se concreta con el establecimiento del Consentimiento Informado, como un documento escrito, diseñado por el profesional de rehabilitación y mejorado con la evidencia clínica. El profesional de rehabilitación también hace uso de la autonomía estableciendo una adecuada relación terapéutica con calidad y calidez. Finalmente la justicia se precisa en el producto de una vida independiente con calidad. Es un modelo de calidad de vida, donde para cada persona puede ser diferente, teniendo en cuenta sus creencias, sus valores, sus intereses, en fin su proyecto de vida. (Figura 1)

Una de las funciones más significativas de un profesional de la salud es el afianzamiento de la relación terapéutica, espacio para el entendimiento de la situación del otro. Analizar la verdadera naturaleza se convierte en una de las principales características durante el proceso de rehabilitación. La relación interactiva que se establece con una o más personas permite conocer y comprender sus sensaciones, emociones y experiencias a través de una comunicación verbal, gestual y corporal.

La relación terapéutica se inicia dentro de un ambiente de confianza y respeto mutuo, partien-



Figura 1

Modelo de rehabilitación basado en la bioética

do de que el ser humano es un ser moral y autónomo, de esta manera se inicia un contacto con el otro donde las reglas deben estar enmarcadas en la claridad de la información, la explicación de lo que sucede, en un lenguaje cotidiano y la capacidad de saber escuchar.

En el contexto de la rehabilitación, la adecuada relación terapéutica es un elemento que trasciende en el objetivo de lograr lo mejor para esa persona, el deseo de hacer el bien (beneficencia), teniendo en cuenta las preferencias del paciente, aspiraciones de cada quien para buscar una vida buena y feliz, una vida con mayor calidad y con menos restricciones. El rehabilitador da alternativas a sus problemas y encuentra en conjunto diferentes estrategias para que la persona aprenda a manejar su necesidad y a satisfacer las demandas del ambiente (autonomía).

El segundo aspecto a tener en cuenta en este modelo es el del Consentimiento Informado en rehabilitación, entendido como derecho, proclama que toda persona aunque esté enferma, con alguna limitación o discapacidad, debe continuar siendo considerada libre y competente para deci- 
dir sobre su integridad y sobre su futuro, por tanto, debe intervenir activamente en las decisiones relacionas con su rehabilitación: aceptar o denegar lo que se le propone después de tener una información oportuna de las alternativas y procedimientos que le van a efectuar.

Es importante exponer también la necesidad de establecer como obligatoriedad en los servicios de rehabilitación el establecimiento de un documento formal y escrito del Consentimiento Informado, para que la persona que presenta la discapacidad o en caso diferente las personas que se encuentren encargadas y la familia, incorporen en el quehacer del rehabilitador el deber de informar acertadamente los procedimientos que se van a realizar con el paciente.

El tercer, y último aspecto que propongo es el resultado de un acertado proceso de rehabilitación a fin de alcanzar la calidad de vida de las personas en situación de discapacidad. La calidad de vida significa de entrada la calidad de la existencia de los seres humanos concretos y reales, en situaciones específicas en las que existen y se esfuerzan por existir, (Maldonado, 2000). La necesidad de vivir se funda en las posibilidades que son, adicionalmente, posibilidades para los demás, posibilidades de grupo y de la comunidad $\mathrm{y}$, en últimas posibilidades de y para la especie.

Según Lukomski (2000), el término calidad de vida entra a formar parte del lenguaje corriente de los países occidentales a partir de 1950 y sólo hasta 1970 adquiere una connotación definida, que se identifica en tres contextos: el descriptivo, el evaluativo o normativo y el prescriptivo o moral. (Tabla 2)

En los últimos años el concepto de Calidad de Vida ha pasado de tener un uso restringido a lo cotidiano, a un empleo más profuso en los más variados ámbitos. De acuerdo a lo investigado ha sido complicado medir empíricamente lo que en forma genérica se fue perfilando como "calidad de vida", es decir, su traducción cuantitativa. Se ha buscado combinar diversos indicadores de bienestar social para establecer índices que permitan comparaciones dentro de cada país en primer lugar, y posteriormente en un conjunto de países.

\section{Tabla 2}

Calidad de vida de la persona con DISCAPACIDAD EN LOS DIFERENTES CONTEXTOS

\begin{tabular}{|l|l|l|}
\hline Contextos & $\begin{array}{l}\text { Persona con } \\
\text { discapacidad }\end{array}$ & Vida con calidad \\
\hline 1. Descriptivo & $\begin{array}{l}\text { Cuál es su } \\
\text { limitación, } \\
\text { cuál es su } \\
\text { discapacidad }\end{array}$ & ¿Qué hace? \\
\hline $\begin{array}{l}\text { 2. Evaluativo o } \\
\text { normativo }\end{array}$ & $\begin{array}{l}\text { Cómo se } \\
\text { encuentra en } \\
\text { este momento } \\
\text { en relación con } \\
\text { su entorno, } \\
\text { su familia y su } \\
\text { grupo social }\end{array}$ & $\begin{array}{l}\text { ¿Qué puede } \\
\text { hacer? }\end{array}$ \\
\hline $\begin{array}{l}\text { 3. Prescriptivo o } \\
\text { moral }\end{array}$ & $\begin{array}{l}\text { Cuáles son sus } \\
\text { nuevas metas, } \\
\text { de acuerdo a } \\
\text { sus intereses y } \\
\text { valores. }\end{array}$ & ¿Qué quiere \\
hacer? \\
¿yándo?
\end{tabular}

Al identificar que la vida con calidad es subjetiva, porque está determinada por la autonomía de cada persona (privada), es indispensable tener en cuenta que el servicio de rehabilitación es un bien común (público), por tanto, lograr para todos iguales oportunidades de desarrollo de sus capacidades, corrigiendo las desigualdades naturales y sociales es la meta del modelo de atención en rehabilitación para la persona con discapacidad, basado en la bioética.

\section{Conclusiones}

Los conceptos aquí expuestos, integran la propuesta teórica de un modelo para la práctica de la atención en rehabilitación de la persona en situa- 
ción de discapacidad, basado en la bioética principialista, brindando una reflexión sobre cada actor, persona con discapacidad, profesional de la rehabilitación y la sociedad. El modelo privilegia el principio de autonomía que por ende se halla intrínsecamente relacionado con el de beneficencia. La persona con discapacidad define autónomamente su sistema de valores, sus objetivos de vida, su propia idea de perfección, de felicidad y de calidad de vida. Lo que es beneficioso para una persona no lo es necesariamente para otra. En este nivel, relacionado con su proyecto de vida, todos somos diferentes, el principio de autonomía, obliga a respetar esa diferencia, dándole prioridad al nivel de lo privado.

El principio de justicia se muestra como el producto de recibir servicios de rehabilitación en forma equitativa y de acuerdo a las necesidades de cada persona, con el objetivo de lograr una vida con calidad en su entorno, es por esto que este modelo podría denominarse EL MODELO DE CALIDAD DE VIDA, dándole un orden social y público, con criterio utilitarista por alcanzar el mayor bien posible, es decir, una vida con calidad para todas las personas con discapacidad, o al menos para la mayoría. Este modelo de atención da énfasis al binomio Autonomía-Justicia.

\section{Referencias}

Angarita Sarmiento, Carlos Enrique. 2000. Estado, poder y derechos humanos en Colombia. Corporación René García. Bogotá, Colombia.

Barahona, Clemente A. 1997. Cáncer infantil: consideraciones éticas y psicológicas. Rev Esp Pediatra 53 (2): 151-61.

BAUER D. 1992. Rehabilitación: enfoque integral principios prácticos. Masson Salvat Medicina Barcelona España, 18.

Boladeras M. 2000. Calidad de vida y principios bioéticos. Colección Bios y Ethos, vol. 15. Ediciones El Bosque.
Botero Uribe, Darío. 2000. El derecho a la utopía. Ecoe Ediciones. Universidad Nacional de Colombia.

Callahan, D. 1990. Tendencias actuales de la ética biomédica en los Estados Unidos de América. Bioética. Organización Panamericana de la Salud. Washington, 168.

CAmps, V. 2001. Una vida de calidad, reflexiones sobre la bioética. Ares y Mares. Editorial Crítica. Barcelona, España.

CASADo D. 1995. Ante la discapacidad, glosas iberoamericanas. Colección Política, Servicios y Trabajo Social. Argentina.

Cely Galindo, G. 1999. La bioética en la sociedad del conocimiento. Colección Bioética. 3R Editores, $187-234$.

Cruz R. 1998. Ponencia presentada en el XIII Seminario Iberoamericano sobre Discapacidad e Información Social. Madrid.

Consejo distrital para las personas que presentan limitaciones de carácter físico, psíquico y sensorial. (200-2005). Comité Técnico Plan Distrital de Discapacidad.

CoRTinA, A. 1995. Ética civil y religión. Madrid. PPC.

Couceiro, A. 1999. Bioética para clínicos. Editorial Tricastela. Madrid. 109-112.

Delgado Díaz, C. Calidad de vida: una perspectiva latinoamericana. Colección Bios y Ethos. Bioética y Calidad de Vida, No. 15, Ediciones El Bosque. Bogotá, Colombia.

Duarte Torres S. 2002. El consentimiento informado en rehabilitación. Revista Colombiana de Rehabilitación, vol. 1, Bogotá

Escobar Triana, J. Comprensión sistémica de la salud y calidad de vida. Colección Bios y Ethos. Bioética y Calidad de Vida, No. 15, Ediciones El Bosque. Bogotá, Colombia.

Escobar Triana, J. La Ley 100 de seguridad social implicaciones bioéticas. Colección Bios y 
Ethos. Bioética y Justicia Sanitaria, No. 9, Ediciones El Bosque. Bogotá, Colombia.

FERrer, J. 1999. Los principios de la bioética. Cuadernos del programa regional de bioética. Organización Panamericana de la Salud \# 7 Universidad del Bosque. Bogotá, Colombia

Galindo, F. 1998. Calidad de vida. Escuela Colombiana de Rehabilitación.

García, G. 1997. La noción de profesión, su ser y su sentido: referentes ético-morales. Colección de Bios y Ethos. Problemas de ética aplicada, No. 4. Ediciones El Bosque. Bogotá, Colombia.

Gracia, D. 2001. La bioética médica en bioética Temas y perspectivas. Organización Panamericana de la Salud, No. 527.

Hotтois, G. 2001. Bioética y derechos humanos. Colección Bios y Ethos. Bioética y derechos $\mathrm{Hu}$ manos. Ediciones El Bosque.

Lesport Esmeral, I. 2003. Sobre la violencia. Colección Bios y Ethos. Bioética y ciencias sociales, vol. 17. Ediciones El Bosque. Bogotá, Colombia.

Lukomski, A. 2000. Calidad de vida: historia y futuro de un concepto problema. Colección Bios y Ethos. Bioética y calidad de vida, No. 15. Ediciones El Bosque. Bogotá, Colombia.

Maldonado. C. 1997. Ética, decisión racional y teoría de la acción. Colección Bios y Ethos. Problemas de ética aplicada, No. 4, Ediciones El Bosque. Bogotá, Colombia.

Maldonado, C. 2000. Fundamentos para la comprensión del problema de la calidad de la vida, mundo, posibilidad y apertura. Colección Bios y Ethos. Bioética y calidad de vida en el siglo XXI, No. 1, Ediciones El Bosque. Bogotá, Colombia.

Méndez GutiérRez, V. 2003. La violencia y el dolor del niño colombiano. Colección Bios y Ethos. Ediciones El Bosque, vol. 17, Bogotá, Colombia.
Ministerio de Salud. 1996. Lineamientos de atención en salud para las personas con deficiencia, discapacidad y/o minusvalía. Bogotá, Colombia.

Ministerio de Salud. 2000. Sistema de garantía de la Calidad para los servicios de salud. Bogotá. Colombia.

Ministerio de Salud. 2000. Discapacidad y costos. Bogotá, Colombia.

Morín, E. 2001. La mente bien ordenada. Editorial Seix Barral S.A. Barcelona 1; 110.

Plan Nacional de Atención a las Personas con Discapacidad. 1999-2002. Andrés Pastrana Arango. República de Colombia.

Presidencia de la República. Política Pública en Discapacidad. Metodología para la Formación. 1998. Consejería Presidencial para la Política Social CPPS, Corporación Andina de Fomento - CAF.

Puig de la Bellacasa, R. 1997. Concepciones, paradigmas y evolución de las mentalidades sobre la discapacidad. Ponencia presentada en el II Seminario sobre Discapacidad e Información, Madrid.

Rodríguez, C. 2001. Calidad de la atención en salud y Ley 100 de seguridad social. Colección Bios y Ethos, No. 9, Ediciones El Bosque, 147-80.

Rueda, L. y Miranda, O. 2001. Principales dilemas bioéticos en las personas con discapacidad prolongada. Revista Chilena de Terapia Ocupacional. Octubre de Universidad de Chile.

SÁnchez Torres, Fernando. 1995. Temas de ética médica. Giro Editores Ltda. Bogotá, Colombia.

SAss, H.M. 1990. La bioética: fundamentos filosóficos y aplicación. Bol of Sanit Panam. 108(5,6): 391-8.

Secretaría Distrital de Salud, Bogotá. D.C. 2001-2005. Plan distrital de discapacidad.

Secretaría Distrital de Salud, Bogotá D.C. (julio de 2001). Lineamientos de atención en salud para las personas con discapacidad en Bogotá, D.C. 
Singer, Peter, Compendio de ética. 1995. Editorial Alianza. Madrid.

The American Journal of Occupational Therapy. 2000. Vol. 54. No. 2, marzo 133.

The American Journal of Occupational Therapy. Vol. 52, No. 3, marzo.
Trujillo Rojas, A. Terapia ocupacional conocimiento y práctica en Colombia. (Julio de 2001). Universidad Nacional de Colombia, primera edición 2002), pág. 355. Las personas con discapacidad en Bogotá, D.C.

Willard/Spackman. 1998. Terapia ocupacional. Ciencia de la Ocupación Humana, 48-50. 